\title{
Stability of VISCOUS flow in a curved channel WITH
} radial temperature gradient

\author{
Sadhana Pandey ${ }^{1}$, A. K. Singh ${ }^{2}$, Neelabh Rai ${ }^{3}$ \\ ${ }^{1}$ Ph.D., Department of Mathematics, Institute of Science, Banaras Hindu University, Varanasi. \\ sadhana.bhu20@gmail.com \\ ${ }^{2}$ Professor, Department of Mathematics, Institute of Science, Banaras Hindu University, Varanasi. \\ ashok@bhu.ac.in \\ ${ }^{3}$ Independent Cyber Security Researcher, CYBER COPS India, Noida. \\ neelabh@cybercops.in
}

\section{ABSTRACT}

In this paper, the stability of Dean's problem in the presence of a radial temperature gradient is studied for narrow gap case. The analytical solution of the eigen value problem is obtained by using the Galerkin's method. The critical values of parameters $\alpha$ and $\Lambda$ are computed, where $\alpha$ is wave number and $\Lambda$ is a parameter determining the onset of stability from the obtained analytical expressions for the first, second and third approximations. It is found that the difference between the numerical values of critical $\Lambda$ corresponding to the second and third approximations is very small as compared to the difference between first and second approximations. The critical values of $\Lambda$ obtained by the third approximation agree very well with the earlier results computed numerically by using the finite difference method. This clearly indicates that for the better result one should obtain the numerical values by taking more terms in approximation. Also, the amplitude of the radial velocity and the cell-patterns are shown on the graphs for different values of the parameter $M$, which depends on difference of temperatures of outer cylinder to the inner one i.e. on $\left(T_{2}-T_{1}\right)$, where $T_{1}$ is the temperature of inner cylinder and $T_{2}$ is the temperature of outer cylinder.

\section{Keywords}

Stability, Radial temperature gradient, Galerkin's method, Curved channel, Narrow gap.

\section{Academic Discipline And Sub-Disciplines}

Fluid Mechanics, Hydrodynamics.

\section{SUBJECT CLASSIFICATION}

\author{
Mathematics
}

\section{INTRODUCTION}

The stability of flow phenomenon of a viscous incompressible fluid between two concentric rotating cylinders with one or both cylinder rotating in the same or opposite directions, was first studied by Taylor [1]. Taylor first found that the shearing motion between concentric cylinders can, under certain conditions, become unstable for small disturbances. The parameter which governs the onset of instability in Taylor problem was called the Taylor number and found that the flow becomes unstable, when the Taylor number exceeds its critical value $T a_{c}$. To determine $T a_{c}$, different methods were given by Taylor [1], Chandrasekhar [2], DiPrima [3], Duty and Reid [4], Harris and Reid [5].This problem was later studied by many researchers because of its practical importance in engineering applications, and is known as the Taylor stability problem.

In the Taylor problem, the stability of the fluid motion is due to the rotational velocity of the cylinders. If now the two concentric cylinders are assumed to be stationary, and the flow is caused by a pressure gradient acting round the curved channel, then the effect of small disturbances on the stability of such a motion, was first studied by Dean [6], and is known as the Dean problem. Dean's analysis is based on a parameter $R \sqrt{d / R_{1}}$, where $\mathrm{R}$ is Reynolds number based on the mean velocity of the fluid. Later, Reid [7] and Hammerlin [8] studied the Dean problem in the narrow-gap case, whereas Walowit et al. [9] studied it for the wide gap case. The eigen value problems in all these papers were solved by using different methods of solution. Dean problem is also solved by Pandey and Prasad [10] for narrow-gap case using Trigometric series method and further the same problem is solved by Prasad and Pandey [11] in the presence of a axial magnetic field.

In all these papers, it was basically assumed that the two cylinders are at the same temperature and as a result of which radial temperature gradient does not exist. However, in many chemical, electrical and mechanical engineering applications the temperature of two cylinders cannot remain the same. Thus, due to the change in the temperature of two cylinders, there exist a temperature gradient and the stability of the fluid flow is affected by the temperature gradient. Hence, Chandrashekhar [12] first studied the effects of the presence of a radial temperature gradient on the onset of instability in the narrow-gap case, by using the method of trigonometric series. The first numerical solution to the Taylor's stability problem in a narrow-gap case was presented by Harris and Reid [5], and this method was later applied to the study of the onset of instability in the presence of a radial temperature gradient by Soundalgekar et al. [13]. The stability of flow in a wide-gap problem was solved by Ali et al. [14] using the finite difference method.

The effects of a radial temperature gradient on the Dean-problem between the narrow-gap annular flow under a pressure gradient acting round the cylinders was studied by Ali et al. [15] using finite difference method. After this the hydrodynamic stability of Taylor-Dean flow between rotating porous cylinders with radial flow is studied by Chang [16] and on a modified Taylor-Dean stability problem where the small gap between the cylinders varies in the azimuthal direction is studied by Eagles [17]. The stability of viscous flow driven by an azimuthal pressure gradient between two porous concentric 
cylinders with radial flow and a radial temperature gradient is studied by Deka et al. [18] and the stability of narrow-gap Taylor-Dean flow with radial heating is studied by Deka and Paul [19]. In engineering applications, the flow driven by both rotating cylinders and an azimuthal pressure gradient can be found. For example, an electro-galvanizing line in the steel making industry uses a roller-type cell to plate zinc onto the surface of a steel strip and a rotating drum filter is used in the paper and board making industry, in which a sheet of fiber is taken off from a drum rotating in a vat full of fiber suspensions.

Here, our aim is to study the narrow-gap Dean-stability problem in the case when the flow is due to the pressure gradient acting round the cylinders. We have solved this problem by using the Galerkin's method and the results are compared with those obtained by Ali et al. [15]. Also, the amplitude of the radial velocity and the cell-pattern are shown on graphs for different values of the parameter $\mathrm{M}$, which depends on difference of temperatures of outer cylinder to the inner one i.e. on $\left(T_{2}-T_{1}\right)$, where $T_{1}$ is the temperature of inner cylinder and $T_{2}$ is the temperature of outer cylinder.

\section{MATHEMATICAL ANALYSIS}

Consider the flow of an incompressible viscous fluid between two concentric cylinders of radii $R_{1}$ and $R_{2}\left(R_{1}\right.$, radius of the inner cylinder; $R_{2}$, radius of the outer cylinder), assuming that the inner and outer cylinders are maintained at two different temperatures $T_{1}$ and $T_{2}$ respectively and flow is due to a constant circumferential pressure gradient.

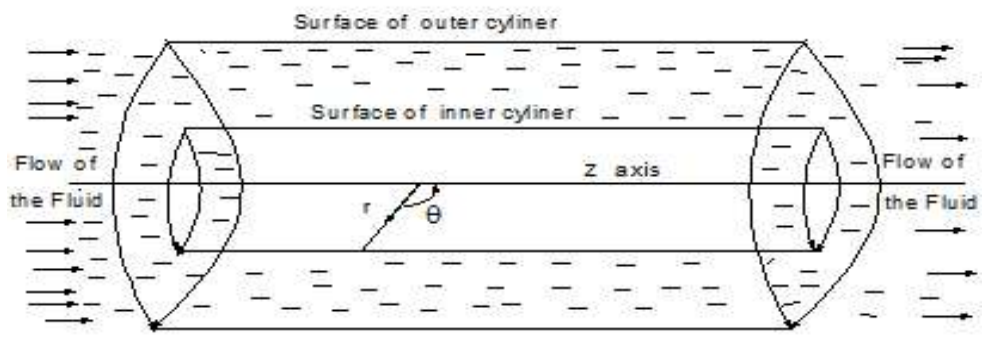

\section{Polar coordinate $(r, \theta$ z) of cylindrical system}

Assuming stationary marginal state, the following differential equations have been obtained to govern the stability of the viscous flow in a narrow-gap annular-space (Ali et al. [15]):

$$
\begin{aligned}
& \left(D^{2}-a^{2}\right)^{2} \bar{u}=\Lambda a^{2}\left[g(x) v-M .(g(x))^{2} \bar{T}\right], \\
& \left(D^{2}-a^{2}\right) v=(1-2 x) \bar{u} \\
& \left(D^{2}-a^{2}\right) \bar{T}=\bar{u} .
\end{aligned}
$$

with boundary conditions

$$
\bar{u}=D \bar{u}=v=\bar{T}=0 \text { at } x=0 \text { and } 1 .
$$

where,

$$
\begin{aligned}
& d=R_{2}-R_{1}, \quad x=\left(r-R_{1}\right) / d, \quad D=\frac{d}{d x}, \quad a=\lambda d, \\
& \xi=r / R_{2}, \quad g(x)=x(1-x), \quad V_{m}=-\left\{d\left(\frac{\partial p}{\partial \theta}\right)\right\} /\left(12 \rho v R_{2}\right), \\
& T=T_{2}-\left(T_{2}-T_{1}\right) \frac{\ln \left(r / R_{2}\right)}{\ln \left(R_{1} / R_{2}\right)}, \quad \bar{T}=\frac{6 T}{\left\{\operatorname{Pr}\left(T_{2}-T_{1}\right)\right\}}, \Lambda=72 \frac{V_{m}{ }^{2} d^{2}}{v^{2} R_{2}}, \\
& M=\frac{1}{2} \alpha \operatorname{Pr}\left(T_{2}-T_{1}\right), \eta=R_{1} / R_{2}, \bar{u}=6(d / v) V_{m} u .
\end{aligned}
$$

Here, $V_{m}$ is the mean velocity across the gap of cylinders.

According to Galerkin's method, we take a sine series for $\bar{T}$ in order to satisfy the boundary conditions given by Eq. (4) as follows:

$$
\bar{T}=\sum_{m=1}^{3} A_{m} \sin (m \pi x) .
$$

Substituting Eq. (6) in Eq. (3) and then with the help of Eq. (2) and (3), we obtain the value of $v$. Using these values of $\bar{T}$ and $v$ in Eq. (1), we have obtained the general solution for $\bar{u}$ as follows:

$$
\begin{aligned}
\bar{u}= & -\Lambda a^{2} \sum_{m=1}^{3} \frac{4 m \pi A_{m}}{K^{3}}\left[\left(A_{1}^{(m)}+x A_{2}^{(m)}\right) \cosh (a x)+\left(A_{3}^{(m)}+x A_{4}^{(m)}\right) \sinh (a x)\right. \\
& -K^{2}\left\{\frac{K_{1} x^{2} \cosh (a x)}{48 a^{4}}+\frac{x^{2}}{24 a^{3}}(2 x-3) \sinh (a x)+K_{2}\left(\frac{K_{1} x^{2} \sinh (a x)}{48 a^{4}}+\right.\right. \\
& \left.\frac{x^{2}}{24 a^{3}}(2 x-3) \cosh (a x)\right)+\frac{1}{4 m \pi K}\left(x \sin (m \pi x)+\frac{4 m \pi \cos (m \pi x)}{K}\right)-\frac{3 K_{3}}{4 m \pi K} \\
& -\frac{1}{K^{2}}\left(x \cos (m \pi x)-\frac{4 m \pi \sin (m \pi x)}{K}\right)+\frac{K_{5}}{2 m \pi K}+\frac{K_{4}}{K^{2}}-\frac{M}{4 m \pi K}\left\{K_{3}+K_{6} .\right.
\end{aligned}
$$


where,

$\left.\left.\left.\sin (m \pi x)+K_{7} \cos (m \pi x)-K_{5}\right\}\right\}\right]$

$$
\begin{aligned}
& K=\left(m^{2} \pi^{2}+a^{2}\right), \quad K_{1}=\left(a^{2} x(2-x)-9\right), \quad K_{2}=\frac{K_{11}}{\sinh (a)}, \\
& K_{3}=x^{2} \sin (m \pi x)+\frac{8 m \pi x \cos (m \pi x)}{K}+\frac{4\left(a^{2}-5 m^{2} \pi^{2}\right) \sin (m \pi x)}{K^{2}}, \\
& K_{4}=x^{2} \cos (m \pi x)-\frac{8 m \pi x \sin (m \pi x)}{K}+\frac{4\left(a^{2}-5 m^{2} \pi^{2}\right) \sin (m \pi x)}{K^{2}}, \\
& K_{5}=x^{3} \sin (m \pi x)+\frac{12 m \pi x^{2} \cos (m \pi x)}{K}+\frac{24 m \pi\left(3 a^{2}-5 m^{2} \pi^{2}\right) \cos (m \pi x)}{K^{3}} \\
& +\frac{12 x\left(a^{2}-5 m^{2} \pi^{2}\right) \sin (m \pi x)}{K^{2}} \\
& K_{6}=\frac{1}{K^{4}}\left(K^{3} x^{2}\left(1+K x^{2}\right)+72 K^{2}\left(1-2 x^{2} m^{2} \pi^{2}\right)-30 m^{2} \pi^{2}\left(5 K-64 m^{2} \pi^{2}\right)\right), \\
& K_{7}=\frac{16 m \pi x}{K^{3}}\left(K^{2} x^{2}+6\left(K-8 m^{2} \pi^{2}\right)\right) \text {, } \\
& K_{8}=1 /\left(\sinh ^{2} a-a^{2}\right), K_{9}=(a \operatorname{coh} a+\sinh a)(-1)^{m}-(a+\sinh a \cosh a) \text {, } \\
& K_{10}=\left(a-(-1)^{m} \sinh a\right), K_{11}=(-1)^{m}-\cosh a \text {, } \\
& A_{1}^{(m)}=1+\frac{4\left(a^{2}-5 m^{2} \pi^{2}\right)}{K^{2}}+\frac{12\left(3 a^{2}-5 m^{2} \pi^{2}\right)}{K^{2}}(1+M), \\
& A_{2}^{(m)}=-(7+2 M)+\frac{1}{K}\left\{4 m^{2} \pi^{2}-3\left(a^{2}-5 m^{2} \pi^{2}\right)-M\left(a^{2}-5 m^{2} \pi^{2}+90\right)\right\} \\
& +\frac{480 M m^{2} \pi^{2} a^{2}}{K^{3}}-a A_{3}^{(m)}, \\
& A_{3}^{(m)}=K_{8}\left[\frac { K ^ { 2 } } { 4 8 a ^ { 4 } } \left\{\left(9-a^{2}\right)(\sinh a \cosh a-a)-2 a \sinh ^{2} a+K_{2}\left(\left(9+a^{2}\right) .\right.\right.\right. \\
& \left.\left.\sinh ^{2} a-2 \operatorname{acosh} a(\sinh a+a \cosh a)\right)\right\}+\frac{\left(a^{2}-5 m^{2} \pi^{2}\right)}{K^{2}}\left(4 K_{9}+3 K K_{10}\right)+ \\
& \frac{12 K_{9}\left(3 a^{2}-5 m^{2} \pi^{2}\right)}{K^{2}}+\left(a(-1)^{m}-\sinh a\right) \cosh a+6 K_{10}-\frac{4 K_{10} m^{2} \pi^{2}}{K}-M \\
& \left\{\frac { 1 } { K } \left(72(-1)^{m} \operatorname{acosh} a-18(-1)^{m} \sinh a+\left(a^{2}-5 m^{2} \pi^{2}\right)\left(5(-1)^{m} \sinh a-\right.\right.\right. \\
& \text { a) } \left.-90 a+36(-1)^{m} m^{2} \pi^{2} \sinh a\right)-\frac{192 m^{2} \pi^{2}(-1)^{m}}{K^{2}}(\operatorname{acosh} a+\sinh a)- \\
& -\frac{12}{K^{3}}\left(K_{9}\left(\left(3 a^{2}-5 m^{2} \pi^{2}\right) K+40 m^{2} \pi^{2} a^{2}\left(a+(-1)^{m} \sinh a\right)\right)-2 a-8\right. \\
& \left.\left.(-1)^{m} \sinh a\right\}\right] \text {, } \\
& A_{4}^{(m)}=\frac{1}{\sinh a}\left[\frac{K^{2}}{48 a^{4}}\left\{\left(a^{2}-9\right)\left(\cosh a+K_{2} \sinh a\right)-2 a\left(\sinh a+K_{2} \cosh a\right)\right\}\right. \\
& +\frac{40 K_{11}}{K^{2}}\left(a^{2}-2 m^{2} \pi^{2}\right)+(-1)^{m}+6 \cosh a+\frac{\cosh a}{K}\left(3 a^{2}-19 m^{2} \pi^{2}\right) \\
& -M\left\{\frac{72(-1)^{m}}{K}-\frac{192 m^{2} \pi^{2}(-1)^{m}}{K^{2}}-\frac{12 K_{11}\left(3 a^{2}-5 m^{2} \pi^{2}\right)}{K^{2}}-\frac{\cosh a}{K}\right. \\
& \left.\left.\left(90-2 K+a^{2}-5 m^{2} \pi^{2}\right)\right\}-A_{3}^{(m)}(\sinh a-\operatorname{acosh} a)\right] .
\end{aligned}
$$

where, $A_{1}^{(m)}, A_{2}^{(m)}, A_{3}^{(m)}, A_{4}^{(m)}$ are the constants of integration, which are obtained using the boundary conditions (4) and solving the resulting equations.

By inserting the mathematical expressions of $\bar{T}$ and $\bar{u}$ from Eqs. (6) and (7) respectively, in Eq. (3), we have,

$$
\begin{aligned}
& \sum_{n=1}^{3} A_{n}\left(n^{2} \pi^{2}+a^{2}\right) \sin (n \pi x)=\Lambda a^{2} \sum_{m=1}^{3} \frac{4 m \pi A_{m}}{K^{3}}\left[\left(A_{1}^{(m)}+x A_{2}^{(m)}\right) \cosh (a x)+\right. \\
& \left(A_{3}^{(m)}+x A_{4}^{(m)}\right) \sinh (a x)-K^{2}\left\{\frac{K_{1} x^{2} \cosh (a x)}{48 a^{4}}+\frac{x^{2}}{24 a^{3}}(2 x-3) \sinh (a x)+K_{2}\right. \\
& \left(\frac{K_{1} x^{2} \sinh (a x)}{48 a^{4}}+\frac{x^{2}}{24 a^{3}}(2 x-3) \cosh (a x)\right)+\frac{1}{4 m \pi K}\left(x \sin (m \pi x)+\frac{4 m \pi \cos (m \pi x)}{K}\right)- \\
& -\frac{3 K_{3}}{4 m \pi K}-\frac{1}{K^{2}}\left(x \cos (m \pi x)-\frac{4 m \pi \sin (m \pi x)}{K}\right)+\frac{K_{5}}{2 m \pi K}+\frac{K_{4}}{K^{2}}-\frac{M}{4 m \pi K}\left\{K_{3}+\right. \\
& \left.\left.\left.K_{6} \sin (m \pi x)+K_{7} \cos (m \pi x)-K_{5}\right\}\right\}\right] .
\end{aligned}
$$

Multiplying Eq. (9) by $\sin (m \pi x)$ and then integrating over the range $0 \leq x \leq 1$, we obtain a system of linear homogeneous equations for the constants and the requirement that these constants are to all zero leads to the following secular equation:

$$
\| \frac{n \pi M_{1}}{R}-\frac{R^{4}}{8 n \pi \Lambda a^{2}} \delta_{m n}-K^{2}\left[\frac{M_{2} \delta_{m n}}{2 m \pi K^{5}}+\frac{M_{3}}{48 a^{4}}+\frac{1}{4 m \pi K}\left(I_{6}-3 I_{8}+2 I_{11}\right)\right.
$$


where,

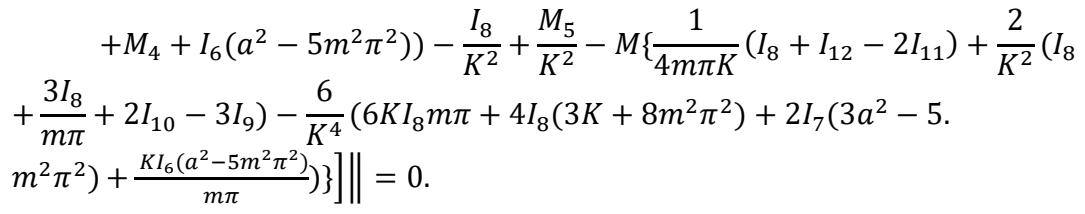

$M_{1}=A_{1}^{(m)}\left(1+(-1)^{n+1} \cosh (a)\right)+A_{3}^{(m)}(-1)^{n+1} \sinh (a)+A_{2}^{(m)}\left((-1)^{n+1}+\right.$ $\left.\left.\left.\frac{2(-1)^{n} a \sinh (a)}{R}\right)+A_{4}^{(m)}\left\{(-1)^{n+1} \sinh (a)+\frac{2 a}{R}\left((-1)^{n} \cosh (a)-1\right)\right)\right\}\right]$,

$M_{2}=K^{2}\left[\left(-3 a^{2}-11 m^{2} \pi^{2}\right)-M\left(a^{2}-5 m^{2} \pi^{2}\right)+18\right]-40 M m^{2} \pi^{2}[7 K-12$. $\left.m^{2} \pi^{2}\right]$

$M_{3}=a^{2}\left(2 I_{5}-I_{13}\right)+a\left(4 I_{2}-3 I_{1}\right)-9\left(I_{3}+I_{1}\right)+K\left(a^{2}\left(2 I_{2}-I_{4}\right)+6 a\left(I_{5}\right.\right.$ $\left.-I_{3}\right)$

$M_{4}=\frac{4}{K^{2}}\left(I_{7}-6\left(I_{8}-I_{9}\right)\right)+\frac{2 I_{7}}{m \pi K^{3}}\left(3 a^{2}-5 m^{2} \pi^{2}\right)$,

$M_{5}=I_{15}+\frac{4 I_{7}\left(a^{2}-5 m^{2} \pi^{2}\right)}{K^{2}}-\frac{8 m \pi I_{6}}{K}$

$M_{6}=\pi^{2}\left(\mathrm{~m}^{2}+\mathrm{n}^{2}\right), M_{7}=\left(1+\mathrm{mn} \pi^{2}\right), M_{8}=\left(1-m n \pi^{2}\right), M_{9}=\left(3+\mathrm{mn} \pi^{2}\right)$,

$M_{10}=\left(3-m n \pi^{2}\right), R=n^{2} \pi^{2}+a^{2}$,

$I_{1}=\frac{\mathrm{n} \pi}{\mathrm{R}}(-1)^{\mathrm{n}}\left[\sinh (\mathrm{a})+\frac{2 \sinh (\mathrm{a})}{\mathrm{R}^{2}}\left(\mathrm{n}^{2} \pi^{2}-3 \mathrm{a}^{2}\right)+\frac{4 \mathrm{a} \cosh (\mathrm{a})}{\mathrm{R}}\right]$,

$I_{2}=\frac{\mathrm{n} \pi}{\mathrm{R}}\left[(-1)^{\mathrm{n}}\left\{-\sinh (\mathrm{a})+\frac{6 \operatorname{acosh}(\mathrm{a})}{\mathrm{R}}+\frac{6 \sinh (\mathrm{a})}{\mathrm{R}^{2}}\left(\mathrm{n}^{2} \pi^{2}-3 \mathrm{a}^{2}\right)+\frac{24 \mathrm{acosh}(\mathrm{a})}{\mathrm{R}^{3}}\right.\right.$ $\left.\left(a^{2}-n^{2} m^{2}\right)\right\}-\frac{24 a}{R^{3}}\left(a^{2}-n^{2} \pi^{2}\right)$,

$I_{3}=\frac{\mathrm{n} \pi}{\mathrm{R}}(-1)^{\mathrm{n}}\left[-\cosh (\mathrm{a})+\frac{2 \cosh (\mathrm{a})}{\mathrm{R}^{2}}\left(\mathrm{n}^{2} \pi^{2}-3 \mathrm{a}^{2}\right)+\frac{4 \operatorname{asinh}(\mathrm{a})}{\mathrm{R}}\right]+\frac{2 \mathrm{n} \pi}{\mathrm{R}^{3}}$ $\left(3 a^{2}-n^{2} \pi^{2}\right)$

$I_{4}=\frac{\mathrm{n} \pi}{\mathrm{R}}(-1)^{\mathrm{n}}\left[-\sinh (\mathrm{a})+\frac{12 \sinh (\mathrm{a})}{\mathrm{R}^{2}}\left(\mathrm{n}^{2} \pi^{2}-3 \mathrm{a}^{2}\right)+\frac{8 \operatorname{acosh}(\mathrm{a})}{\mathrm{R}}+\frac{96 \cosh (\mathrm{a})}{\mathrm{R}^{3}}\right.$ $\left.\left(a^{2}-n^{2} \pi^{2}\right)+\frac{24 \sinh (a)}{R^{4}}\left\{n^{4} \pi^{4}-5 a^{2}\left(a^{2}-2 n^{2} \pi^{2}\right)\right\}\right]$,

$I_{5}=\frac{n \pi}{R}(-1)^{n}\left[-\cosh (a)+\frac{24 a \sinh (a)}{R^{3}}\left(a^{2}-n^{2} \pi^{2}\right)+\frac{6 a \sinh (a)}{R}+\frac{6 \cosh (a)}{R^{2}}\right.$. $\left.\left(n^{2} \pi^{2}-3 a\right)\right]$,

$I_{6}= \begin{cases}\frac{1}{4} ; & \text { if } m=n \\ \frac{\left.2 \mathrm{mn}\left((-1)^{\mathrm{m}+\mathrm{n}}-1\right)\right)}{\pi^{2}\left(\mathrm{~m}^{2}-\mathrm{n}^{2}\right)^{2}} ; & \text { if } m \neq n\end{cases}$

$\delta_{m n}= \begin{cases}1 ; & \text { if } m=n \\ 0 ; & \text { if } m \neq n\end{cases}$

$\begin{cases}0 ; & \text { if } m=n\end{cases}$

$I_{7}= \begin{cases}\frac{\left.\mathrm{n}\left((-1)^{\mathrm{m}+1}-1\right)\right)}{\pi\left(\mathrm{m}^{2}-\mathrm{n}^{2}\right)} ; & \text { if } m \neq n\end{cases}$

$I_{8}= \begin{cases}-\frac{1}{4 m \pi} ; & \text { if } m=n \\ \frac{\mathrm{n}(-1)^{\mathrm{m}+\mathrm{n}}}{\pi\left(\mathrm{m}^{2}-\mathrm{n}^{2}\right)} ; & \text { if } m \neq n\end{cases}$

$I_{9}= \begin{cases}-\frac{1}{4 m \pi} ; & \text { if } m=n \\ \frac{1}{2 \pi^{3}}\left[\frac{2+(-1)^{\mathrm{m}-\mathrm{n}}\left(M_{6}-2 M_{7}\right)}{(\mathrm{m}-\mathrm{n})^{3}}-\frac{2+(-1)^{\mathrm{m}+\mathrm{n}}\left(M_{6}-2 M_{8}\right)}{(\mathrm{m}+\mathrm{n})^{3}}\right] ; & \text { if } m \neq n\end{cases}$

$I_{10}= \begin{cases}\frac{3}{8 m^{3} \pi^{3}}-\frac{1}{4 m \pi} ; & \text { if } m=n \\ \frac{1}{2 \pi^{3}}\left[\frac{(-1)^{\mathrm{m}-\mathrm{n}}\left(M_{6}-2 M_{9}\right)}{(\mathrm{m}-\mathrm{n})^{3}}-\frac{(-1)^{\mathrm{m}+\mathrm{n}}\left(M_{6}-2 M_{10}\right)}{(\mathrm{m}+\mathrm{n})^{3}}\right] ; & \text { if } m \neq n\end{cases}$

$I_{11}= \begin{cases}\frac{1}{8}\left(1-\frac{3}{\mathrm{~m}^{2} \pi^{2}}\right) ; & \text { if } m=n \\ \frac{3}{2 \pi^{4}}\left[\frac{2+(-1)^{\mathrm{m}-\mathrm{n}}\left(M_{6}-2 M_{7}\right)}{(\mathrm{m}-\mathrm{n})^{4}}-\frac{2+(-1)^{\mathrm{m}+\mathrm{n}}\left(M_{6}-2 M_{8}\right)}{(\mathrm{m}+\mathrm{n})^{4}}\right] ; & \text { if } m \neq n\end{cases}$ 


$$
\begin{aligned}
& I_{12}= \begin{cases}\frac{1}{10}+\frac{3-2 \mathrm{~m}^{2} \pi^{2}}{4 \mathrm{~m}^{4} \pi^{4}} ; & \text { if } m=n \\
\frac{2}{\pi^{4}}\left[\frac{(-1)^{\mathrm{m}-\mathrm{n}}\left(M_{6}-2 M_{9}\right)}{(\mathrm{m}-\mathrm{n})^{4}}-\frac{(-1)^{\mathrm{m}+\mathrm{n}}\left(M_{6}-2 M_{10}\right)}{(\mathrm{m}+\mathrm{n})^{4}}\right] ; & \text { if } m \neq n\end{cases} \\
& I_{13}=\frac{1}{\mathrm{R}^{5}}\left[24\left\{5 \mathrm{a}^{2} \mathrm{n} \pi\left(\mathrm{a}^{2}-2 \mathrm{n}^{2} \pi^{2}\right)+\mathrm{n}^{5} \pi^{5}\right\}-\mathrm{n} \pi(-1)^{\mathrm{n}} \cosh (\mathrm{a})\left\{\mathrm { a } ^ { 6 } \left(\mathrm{a}^{2}+4(9+\right.\right.\right. \\
& \left.\left.n^{2} \pi^{2}\right)\right)+n^{4} \pi^{4}\left(24-12 n^{2} \pi^{2}+n^{4} \pi^{4}\right)+4 a^{2} n^{2} \pi^{2}\left(-60+3 n^{2} \pi^{2}+\right. \\
& \left.\left.n^{4} \pi^{4}\right)+6 a^{4}\left(20+10 n^{2} m^{2}+n^{4} \pi^{4}\right)\right\}+8 n \pi a(-1)^{n}\left\{a^{6}+3 a^{2} n^{4} \pi^{4}\right. \\
& \left.\left.+n^{4} \pi^{4}\left(-12+n^{2} \pi^{2}\right)+3 a^{4}\left(4+n^{2} \pi^{2}\right)\right\} \sinh (a)\right] \text {. }
\end{aligned}
$$

On substituting the values of $\mathrm{A}_{1}{ }^{(\mathrm{m})}, \mathrm{A}_{2}{ }^{(\mathrm{m})}, \mathrm{A}_{3}{ }^{(\mathrm{m})}, \mathrm{A}_{4}{ }^{(\mathrm{m})}$ and $\mathrm{I}_{1}, \mathrm{I}_{2}, \mathrm{I}_{3} \ldots . \mathrm{I}_{12}, \mathrm{I}_{13}$ from Eq. (8) and (11) in Eq. (10) and taking $M=0$ we have,

$$
\begin{aligned}
& \| \frac{m n \pi^{2}}{R}\left[\frac{4}{K}\left\{(-1)^{n}\left(\frac{4 \mathrm{a} \sinh (a)}{R}-\cosh (a)\right)-1\right\}-R_{1} K_{8}\left\{(-1)^{n+1} \sinh (a)-\right.\right. \\
& \left.\frac{4 \mathrm{a}\left(\cosh (a)+(-1)^{n+1}\right)}{R}\right\}-R_{2} K_{8}\left\{(-1)^{n+1}\left(\frac{6 \mathrm{a} \sinh (a)}{R}-\cosh (a)\right)+\right. \\
& \left.\frac{4\left(\mathrm{n}^{2} \mathrm{~m}^{2}-3 \mathrm{a}^{2}\right)}{\mathrm{R}^{2}}\left(1+(-1)^{n+1} \cosh (a)\right)\right\}+R_{3} K_{8}\left\{\left(\frac{6 \mathrm{a} \cosh (a)}{R}-\sinh (a)\right)-\frac{2 a}{R}\right. \\
& \left.\left.+\frac{4\left(\mathrm{n}^{2} \pi^{2}-3 \mathrm{a}^{2}\right)}{\mathrm{R}^{2}}(-1)^{n+1} \sinh (a)\right\}\right]+R_{4}+\frac{4 R_{5}\left(5 \mathrm{~m}^{2} \pi^{2}-\mathrm{a}^{2}\right)}{\mathrm{K}^{2}}+\frac{4 R_{6} m \pi}{\mathrm{K}}+ \\
& \frac{R^{4}}{8 n \pi \Lambda a^{2}} \delta_{m n} \|=0 .
\end{aligned}
$$

where,

$$
\begin{aligned}
& R_{1}=A a+B(\sinh (a)+\operatorname{acosh}(a))-C \sinh (a), \\
& R_{2}=A \sinh ^{2}(a)+a B(\sinh (a)+\operatorname{acosh}(a))-C \operatorname{asinh}(a) \text {, } \\
& R_{3}=A(\sinh (a) \cosh (a)-a)+B a^{2} \sinh (a)-C(\operatorname{acosh}(a)-\sinh (a)) \text {, } \\
& R_{4}=\left\{\begin{array}{lc}
\frac{12}{\pi^{4}} \frac{(m+n)^{4}-(m-n)^{4}}{\left(\mathrm{~m}^{2}-\mathrm{n}^{2}\right)}-\frac{4 m n}{\pi^{2}\left(\mathrm{~m}^{2}-\mathrm{n}^{2}\right)^{2}} ; & \text { if } m+n \text { is odd } \\
0 ; & \text { otherwise }
\end{array}\right. \\
& R_{5}= \begin{cases}\frac{8}{\pi^{3}} \frac{(m+n)^{4}-(m-n)^{4}}{\left(\mathrm{~m}^{2}-\mathrm{n}^{2}\right)^{3}}+\frac{2 n}{\pi\left(\mathrm{m}^{2}-\mathrm{n}^{2}\right)} ; & \text { if } m+n \text { is odd } \\
0 ; & \text { otherwise }\end{cases} \\
& R_{6}=\left\{\begin{array}{l}
\frac{8 m n}{\pi^{2}\left(\mathrm{~m}^{2}-\mathrm{n}^{2}\right)^{2}} \\
0 ;
\end{array}\right. \\
& \text { if } m+n \text { is odd } \\
& A=\frac{12\left(m^{2} \pi^{2}-a^{2}\right)}{K^{2}}, B=\frac{4(-1)^{m}-\cosh a}{K}, C=(-1)^{m+1} A+\frac{4 \operatorname{asinh}(a)}{K},
\end{aligned}
$$

Eq. (12) is the result which is obtained by Chandrashekhar [20].

\section{RESULT AND DISCUSSION}

The numerical value of $\Lambda_{c}$ computed from Eq. (10) corresponding to the first, second and third approximations are listed in Table 1. In this table $\Lambda_{c_{1}}, \Lambda_{c_{2}}, \Lambda_{c_{3}}$ represent the numerical values corresponding to the first, second and third approximations, while $\Lambda_{c}$ is the values obtained by Ali et al. [15]. This table clearly shows that the values of $\Lambda_{c}$ obtained by the third approximation agree very well with the values obtained by Ali et al. [15] using the finite difference method.

From Table 1 we find that, when $M=0$ (ie. when the two cylinders are at same temperature), then the value of $\Lambda_{c}$ is very similar to that value which was computed by Walowit et al. [9]. For positive values of $\mathrm{M}$ (i.e. when the temperature of outer cylinder is higher than that of inner cylinder), the values of $\Lambda_{c}$ are less than those for $M=0$, which shows that the flow becomes unstable and hence flow gets destabilized owing to increasing +ve values of $\mathrm{M}$. The reason behind this is that when the temperature of outer cylinder is higher than that of inner cylinder, the denser fluid is nearer to the inner cylinder, then the centrifugal effect tends to drive the denser fluid in to the gap and this will promote the instability. On the other hand when $\mathrm{M}$ is -ve (i.e. outer cylinder is cooler than that of inner cylinder), the value of $\Lambda_{c}$ increases and hence flow gets stabilized owing to increasing -ve values of $\mathrm{M}$. The reason behind this is that when the temperature of inner cylinder is higher than that of outer cylinder, the denser fluid is nearer to the outer cylinder and therefore higher values of $\Lambda_{c}$ are required to overcome the stabilizing centrifugal effect.

Table 1. Value of critical Dean and wave numbers 


\begin{tabular}{|c|c|c|c|c|c|c|}
\hline $\boldsymbol{M}$ & $\boldsymbol{a}_{\boldsymbol{c}}$ & $\boldsymbol{\Lambda}_{\boldsymbol{c}_{1}}$ & $\boldsymbol{\Lambda}_{\boldsymbol{c}_{2}}$ & $\boldsymbol{\Lambda}_{\boldsymbol{c}_{3}}$ & $\boldsymbol{\Lambda}_{\boldsymbol{c}}$ & $\begin{array}{c}\boldsymbol{\Lambda}_{\boldsymbol{c}} \text { computed } \\
\text { by Walowit et } \\
\text { al. }\end{array}$ \\
\hline-1.0 & 5.029 & 333572 & 333583 & 333591 & 333592 & \\
\hline-0.75 & 4.844 & 244169 & 244178 & 244178 & 244180 & \\
\hline-0.5 & 4.582 & 178196 & 178198 & 178206 & 178213 & \\
\hline-0.25 & 4.284 & 129145 & 129169 & 129172 & 129185 & \\
\hline 0 & 3.950 & 92766 & 92776 & 92778 & 92782 & 92794 \\
\hline 0.25 & 3.639 & 66598 & 66645 & 66668 & 66669 & \\
\hline 0.5 & 3.42 & 49078 & 49088 & 49087 & 49085 & \\
\hline 0.75 & 3.302 & 37643 & 37652 & 37658 & 37657 & \\
\hline 1.0 & 3.235 & 30101 & 30104 & 30105 & 30107 & \\
\hline
\end{tabular}

Other interesting phenomenon is to know the behaviour of the amplitude of the radial velocity and the corresponding cellpattern. So for a set of values of $a_{c}$ and $\Lambda_{c}$, the values of $A_{2}^{(m)} / A_{1}^{(m)}, A_{3}^{(m)} / A_{1}^{(m)}$ are determined from Eq. (4). The eigenfunctions thus obtained are normalised so that the amplitude of the radial component of the velocity perturbation is unity. These eigenfunctions $\mathrm{u}(\mathrm{x})$ and the corresponding cell-pattern for the stream function $\Psi=\mathrm{u}(\mathrm{x}) \cdot \cos \left(a_{c} \mathrm{z}\right)$ at the onset of instability for different values of $\mathrm{M}$ are shown in Figs. 1-5.

In Fig. 1, when $\mathrm{M}$ is -ve i.e. outer cylinder is cooler then the inner cylinder, the cells are circular in the annular passage between the cylinders, because in this case the centrifugal effect is acting on temperature gradient and this confirms the stabilization of flow when $\mathrm{M}$ is -ve. In Fig. 2, when $\mathrm{M}=0$, i.e. both the cylinders are at same temperature, then the cells patterns are not circular and are elliptical having small difference in major and minor axes.

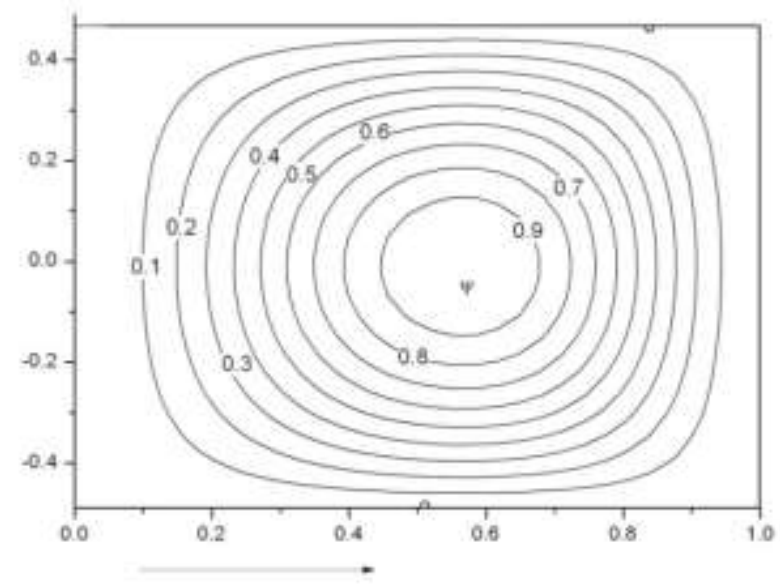

Fig 1: The cell pattern at the onset of instability for $M=-0.75$ 


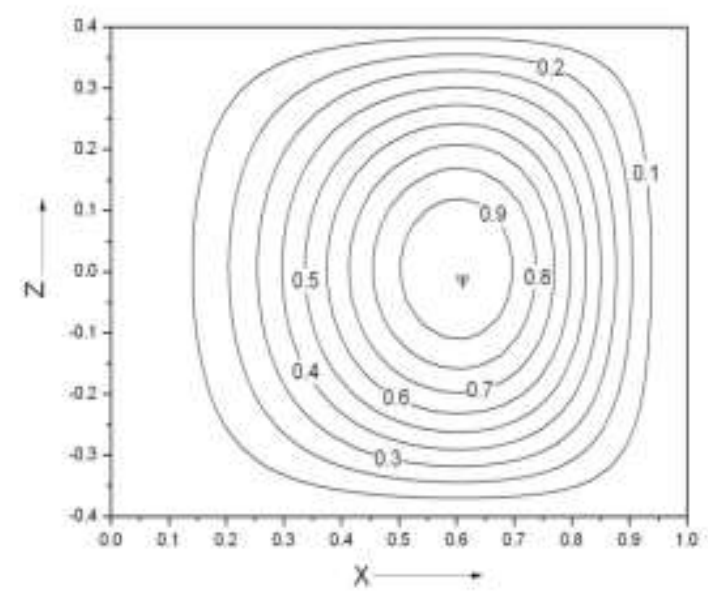

Fig 2: The cell pattern at the onset of instability for $M=0.0$

In Fig. 3, when $\mathrm{M}=0.5$, we see that cells are broken in to two parts in which one is in clockwise direction, while other is in anticlockwise direction. When we compare the Fig. 3 and Fig. 4, we find that the cells are shifted towards the inner cylinder, because in this case the convection currents are flowing from outer cylinder towards the inner one. The formation of corners along the edges of the cells is seen near the inner cylinder and the cells will start breaking near the inner cylinder through these corners. This confirms the destabilization of flow when $\mathrm{M}$ is $+\mathrm{ve}$ and it become more and more unstable as we increase the +ve value of $\mathrm{M}$.

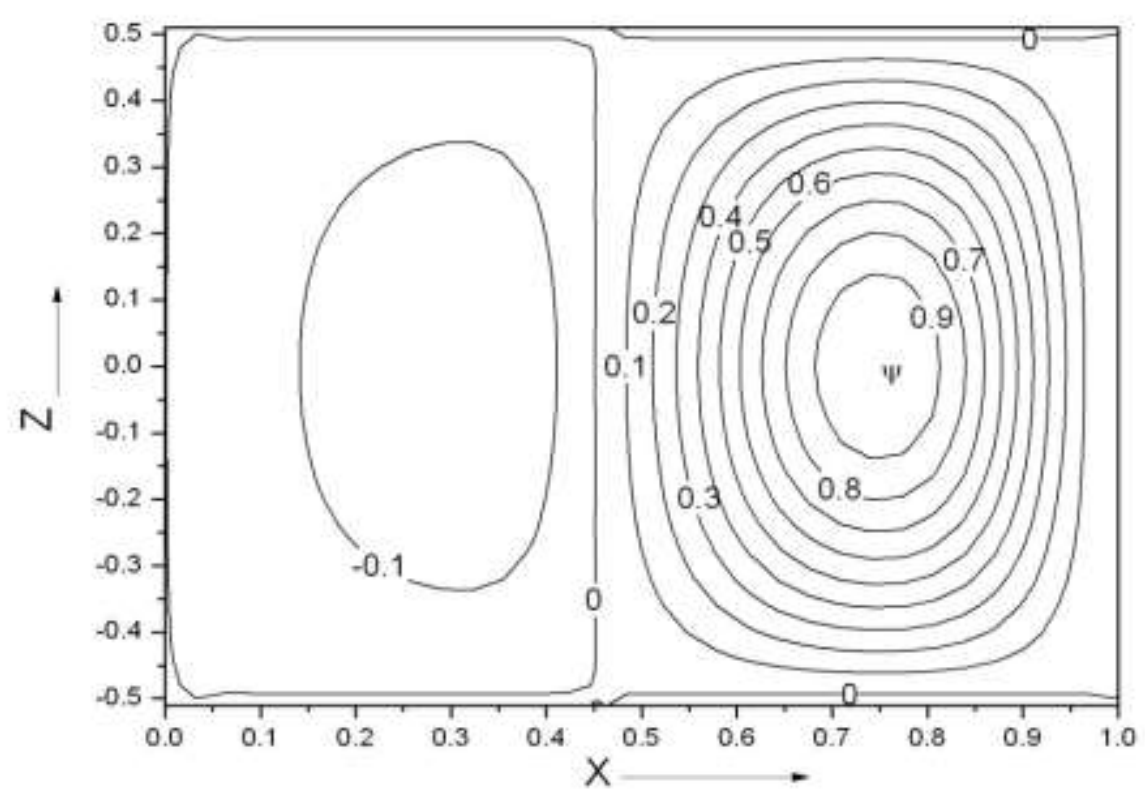

Fig 3: The cell pattern at the onset of instability for $M=0.5$ 


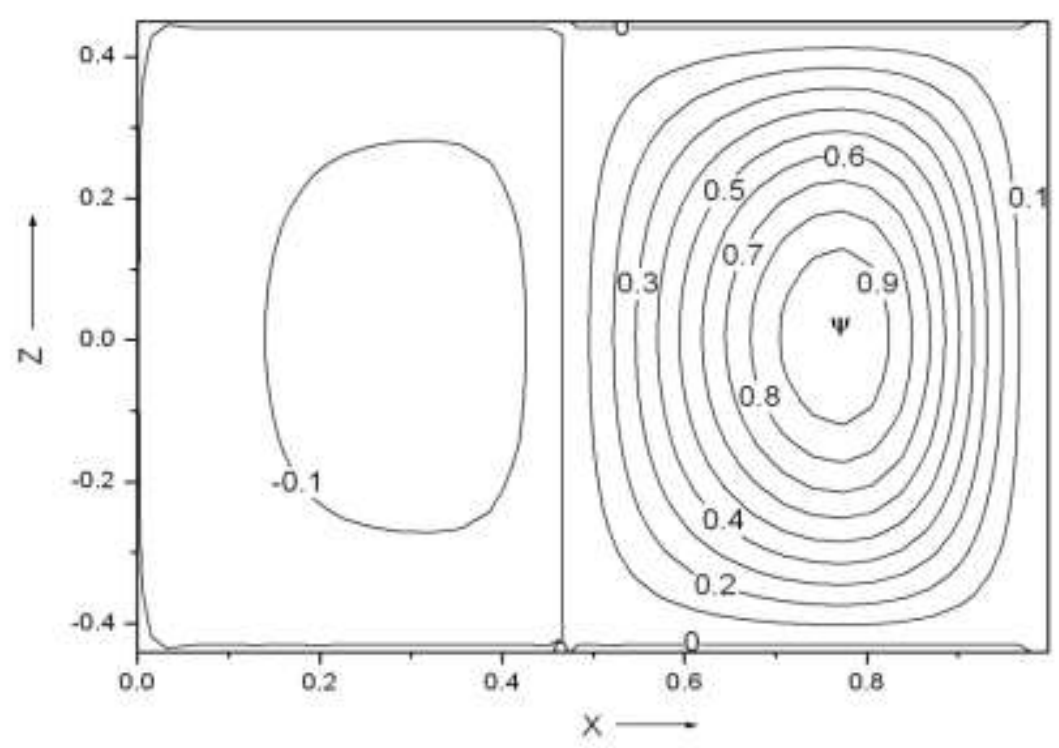

Fig 4: The cell pattern at the onset of instability for $M=0.75$

From Fig. 5 we find that the maximum of $u(x)$ shifts toward the inner cylinder, in the case when $M$ is +ve as compared to the case of $M=0$, whereas in the case when $\mathrm{M}$ is -ve the maximum of $\mathrm{u}(\mathrm{x})$ shifts toward the outer cylinder. And as we increase the +ve values of $M$, the maximum of $u(x)$ shifts more and more toward the inner cylinder.

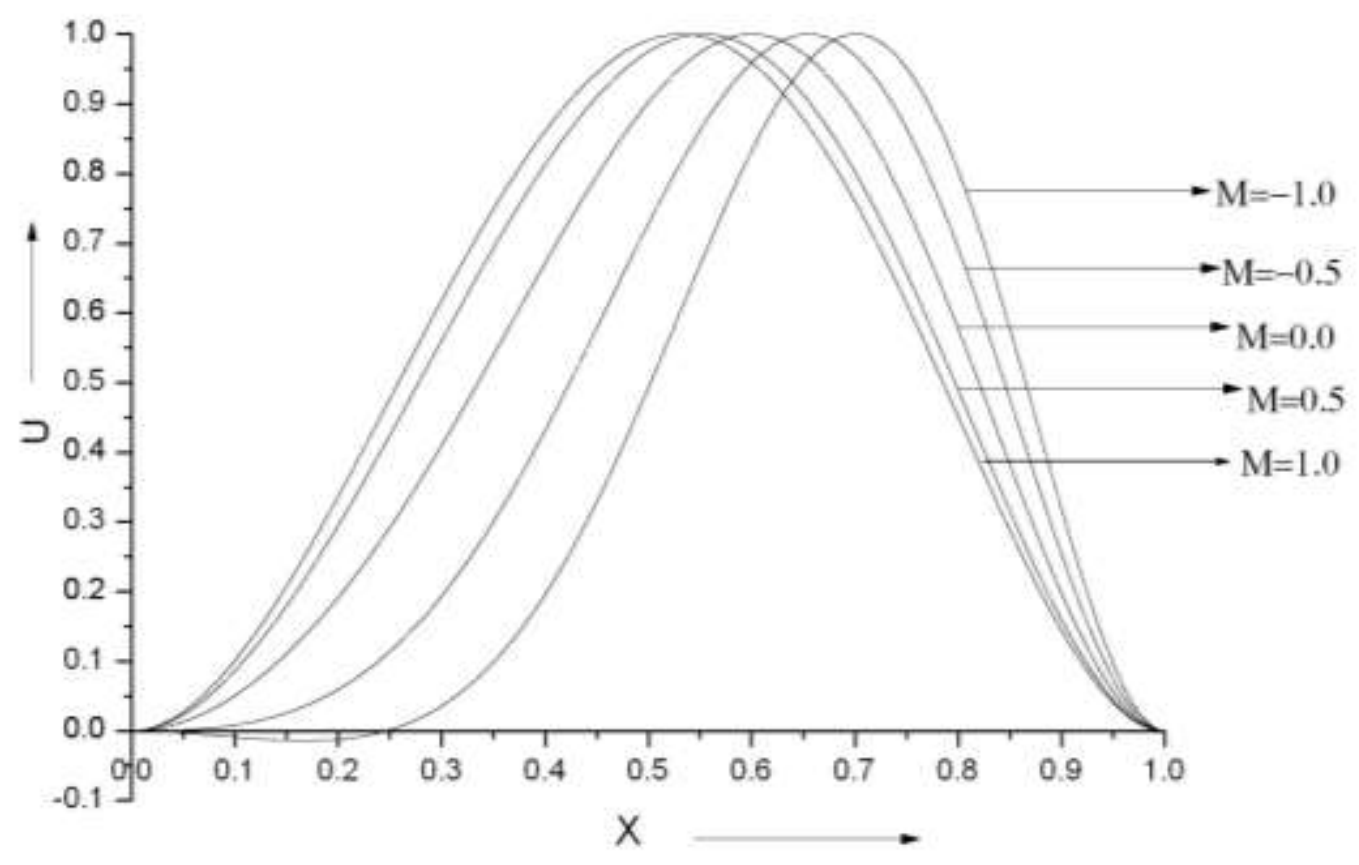

Fig 5: The radial velocity perturbation $u(x)$ at the onset of instability.

\section{CONCLUSION}

The channel flow is more stable when the temperature of inner cylinder is higher than that of outer cylinder (i.e. $\mathrm{M}$ is $-\mathrm{ve}$ ). Hence, the flow inside the annular passage of two concentric cylinders can be maintained in laminar state by raising the temperature of inner cylinder higher than that of outer cylinder. This conclusion is most important from the point of view of industrial applications. The fluid flow gets destabilized owing to increasing the +ve values of $M$ (i.e. the temperature of outer cylinder is less than that of inner cylinder). In the case, when the temperature of inner cylinder is less than that of outer cylinder, the maximum of $\mathrm{u}(\mathrm{x})$ shifts toward the inner cylinder as compared to the case, when the temperatures of 
both the cylinders are same, whereas the case, when the temperature of outer cylinder is less than that of inner cylinder the maximum of $\mathrm{u}(\mathrm{x})$ shifts toward the outer cylinder.

\section{Nomenclature}

$\begin{array}{ll}\mathrm{a} & \text { Dimensionless wave number } \\ \mathrm{r} & \text { Distance from the axis of cylinder } \\ \mathrm{d} & \text { Difference between two radii of the cylinders } \\ R_{1}, R_{2} & \text { Radii of inner and outer cylinders respectively } \\ u, v, w & \text { Velocity components in } \mathrm{r}, \theta \text { and } \mathrm{z} \text { directions respectively } \\ \mathrm{K} & \text { Thermal conductivity } \\ \mathrm{Pr} & \text { Prandtl number } \\ \Lambda & \text { Parameter determining the onset of instability } \\ T_{1}, T_{2} & \text { Temperature of inner and outer cylinder respectively. }\end{array}$

\section{Greek symbols}

$\rho \quad$ Density of fluid

v Kinematic Viscosity

$\eta \quad$ Ratio of radii of cylinders $\left(R_{1} / R_{2}\right)$

\section{REFERENCES}

[1] Taylor, G. 1923. Stability of a viscous liquid contained between two rotating cylinders. Philosophical Transactions of the Royal Society A 223, 289-343.

[2] Chandrasekhar, S. 1953. The stability of viscous flow between rotating cylinders. Mathematica 1, 5-13.

[3] DiPrima, R. (1955). Application of the Galerkin method to the calculation of the stability of curved flows. Quartely Applied Mathematics 13, 55-62.

[4] Dutty, R.L. and Reid, W.H. 1964. On the stability of viscous flow between rotating cylinders, Part 1, Asymptotic Analysis. Journal of Fluid Mechanics 20, 81-94.

[5] Harris, D.L. and Reid, W.H. 1964. On the stability of viscous flow between rotating cylinders, Part 2, Numerical Analysis. Journal of Fluid Mechanics 20, pp 95-101.

[6] Dean, W.R. 1928. Fluid motion in a curved channel. Proceedings of the Royal Society of London A 121, $402-420$.

[7] Reid, W.H. 1958. On the stability of viscous flow in a curved channel. Proceedings of the Royal Society of London A 244, 186-198.

[8] Hammerlin, C. 1958. Die stabilität derstromung in einem Gekrummten Kanel. Arch. Rational Mechanical analysis 1, 212-224.

[9] Walowit, J.S., Tsao and DiPrima, R.C. 1964. Stability of flow between arbitrarily spaced concentric cylindrical surfaces including the effects of temperature gradient. Transactions of ASME Journal of Applied Mechanics 31, 585-593.

[10] Pandey, S. and Prasad, R. 2012. Stability of a pure pressure maintained flow in a curved channel. Proceeding of Mathematical Society, B.H.U. 28, 49-53.

[11] Prasad, R. and Pandey, S. 2012. Stability of a pure pressure maintained flow in a curved channel in presence of an axial magnetic field. Proceeding of Mathematical Society, B.H.U. 28, 43-48.

[12] Chandrasekhar, S. 1954. The stability of flow between rotating cylinders in the presence of radial temperature gradient. Journal of Rational Mechanics and Analysis 3, 181-207.

[13] Soundalgekar, V.M., Takhar, H.S. and Smith, T.J. 1981. Effect of radial temperature gradient of viscous flow in an annulus with a rotating inner cylinder. Wärme-Stoffübertrag 15, 233-238.

[14] Ali, M. A., Soundalgekar, V. M. and Takhar, H.S. 1995. Finite difference analysis of Stability of flow in a curved channel-wide gap problem. Arch. Mechanics 47, 3-11.

[15] Ali, M.A., Takhar, H..S and Soundalgekar, V.M. 1998. Effect of radial temperature gradient on the stability of flow in a curved-narrow gap problem. Proceedings of the Royal Society of London A 454, 2279-2287. 
[16] Chang, M.H. 2003. Hydrodynamic stability of Taylor-Dean flow between rotating porous cylinders with radial flow. Physics of Fluids 15(5), 1178-1188.

[17] Eagles, P.M. 2004. On a modified Taylor-Dean stability problem where the small gap between the cylinders varies in the azimuthal direction. Physics of Fluids 16, 546-550.

[18] Deka, R.K., Gupta, A.S. and Das, S.K. 2007. Stability of viscous flow driven by an azimuthal pressure gradient between two porous concentric cylinders with radial flow and a radial temperature gradient. Acta Mechanica 189, 73-86.

[19] Deka, R.K. and Paul, Ashish 2013. The Stability of narrow-gap Taylor-Dean flow with radial heating: Stationary critical modes. Computer and Fluids 82, 87-94.

[20] Chandrasekhar, S (1961): Hydrodynamic and hydromagnetic stability, Oxford University Press, London.

\section{Author' biography with Photo}

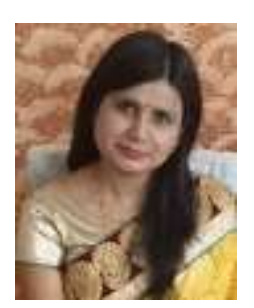

Dr. Sadhana Pandey had completed her M.Sc. \& Ph.D. in Mathematics from Banaras Hindu University (B.H.U), Varanasi, India in 2010 \& 2015 respectively. Her research area is Fluid Mechanics and currently working on Hydrodynamics. She is currently the lifetime member of the Mathematical Society, B.H.U, India. She had authored four research papers in reputed international \& national journals. She had worked as an Assistant Professor in Mathematics at CMR Engineering College, Hyderabad, India.
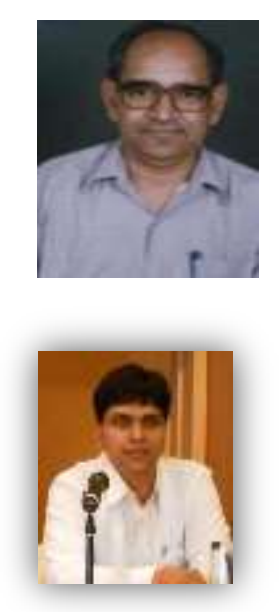

Dr. A. K. Singh is working as a Professor in the Department of Mathematics, Banaras Hindu University (B.H.U), Varanasi, India. He earned his Ph.D. in Mathematics \& D.Sc. in Mathematics from B.H.U, Varanasi, India in 1979 \&1986 respectively. His research interest includes Differential Equations, Fluid Dynamics, Numerical Analysis, Magnetohydrodynamic Flows, Flow through porous media, NonNewtonian fluids, Heat and Mass transferand Transport processes in cavity. He is currently the president of Mathematical Society, B.H.U, India. He has authored and co-authored 186 papers in the reputed international \& national journals. 2011 towards cyber security, SCADA Security, and research work under the banner of CYBER COPS India. He is a member of numerous societies viz. Internet Society, IETE, DSCI, ICTTF, Association of Old Crows, etc.

Mr. Rai is an Engineering Graduatein IT (2008), and Diploma in Indian cyberlaw (2008). He is a perpetual new goal setter, who goes on adding exciting objectives. He is a Certified in many certifications viz. Certified Cyber Crime Investigator (2009) \& Certified Cyber Warfare \& Defence Professional (2010), an EC-Council Certified Incident Handler (2012), a BSI Certified Risk Management Implementer (2013), IRCA Certified Information Security Management System Lead Auditor [ISO 27001:2015] (2014) \& IRCA Certified Business Continuity Management System Lead Auditor [ISO 22301:2012] (2015). 